\title{
Reflets
}

Revue ontaroise d'intervention sociale et communautaire

\section{Dynamique de l'aide informelle auprès des personnes âgées}

\section{Linda Ladouceur}

Volume 2, numéro 2, automne 1996

Vieillir à l'aube de l'an 2000

URI : https://id.erudit.org/iderudit/026132ar

DOI : https://doi.org/10.7202/026132ar

Aller au sommaire du numéro

Éditeur(s)

Reflets : Revue ontaroise d'intervention sociale et communautaire

ISSN

1203-4576 (imprimé)

1712-8498 (numérique)

Découvrir la revue

Citer cet article

Ladouceur, L. (1996). Dynamique de l'aide informelle auprès des personnes âgées. Reflets, 2(2), 100-108. https://doi.org/10.7202/026132ar

Tous droits réservés (C) Reflets : Revue ontaroise d'intervention sociale et communautaire, 1996
Ce document est protégé par la loi sur le droit d'auteur. L'utilisation des services d'Érudit (y compris la reproduction) est assujettie à sa politique d'utilisation que vous pouvez consulter en ligne.

https://apropos.erudit.org/fr/usagers/politique-dutilisation/ 


\section{Dynamique de l'aide informelle auprès des personnes âgées}

L'opinion exprimée dans cet article est celle de l'auteure et ne réflète pas nécessairement les points de vue ou les politiques de Santé Canada ou du gouvernement du Canada.

\section{Linda Ladouceur}

Analyste principale en évaluation de programmes, Santé Canada, Ottawa

\section{Introduction}

"...le maintien à domicile des personnes agées est de plus en plus prôné comme une façon de réduire les dépenses de l'État au niveau des soins de santé et des services sociaux.»
Dans les pays industrialisés, le maintien à domicile des personnes âgées est de plus en plus prôné comme une façon de réduire les dépenses de l'État au niveau des soins de santé et des services sociaux. Cette direction n'est cependant pas sans avoir des répercussions pour les familles qui offrent déjà de 85 à $90 \%$ des soins dont bénéficient les personnes âgées (CCNTA, 1989:4). Les nombreux écrits publiés à ce jour sur l'aide informelle ont d'ailleurs amplement documenté le fardeau que peut représenter un tel accompagnement. Par contre, peu de recherches ont essayé de comprendre le sens que prend ce geste dans la vie des aidantes et des aidants informels.

Cet article présente quelques résultats d'une recherche exploratoire de type qualitatif, que nous avons menée sur la signification du geste d'accompagnement d'une personne âgée (Ladouceur, 1995). Ces résultats proviennent: (a) d'une analyse documentaire, (b) d'une observation participante au sein de deux programmes de jour pour personnes âgées ayant des limites physiques ou cognitives, et (c) d'entrevues semi-dirigées menées auprès de quatre aidants informels. Nous concluons l'analyse des résultats ainsi obtenus par une réflexion sur les implications pour la pratique et les politiques sociales. 


\section{Problématique de l'aide informelle}

"Les aidantes et aidants informels font toutes sortes de choses.»
Voyons d'abord en quoi consiste la réalité de l'aide informelle.

Elle est offerte surtout par les femmes, et cela, dans $70 \%$ à $80 \%$ des cas (Lesemann et Martin, 1993 : 207). Ce sont d'abord les épouses, mais aussi les filles et les belles-filles des personnes âgées frêles (Brody 1990: 4).

L'âge des aidantes et aidants varie de 30 à 80 ans (CCNTA 1989), la moyenne se situant à un peu moins de 60 ans (Guberman et al. 1993; Lesemann et Chaume 1989). Enfin, selon l'étude de Paquet (1992), la majorité (86 \%) ne travaille pas à l'extérieur du foyer.

\section{Tâches et répercussions}

Les aidantes et aidants informels font toutes sortes de choses. Ils offrent de l'aide à la personne qu'ils accompagnent au niveau de l'hygiène personnelle, de l'habillage, des repas, et de l'entretien ménager. Mais là ne s'arrête pas leur travail. Ils offrent également du soutien psychologique ou émotif, de l'assistance financière, ou encore agissent comme médiateurs entre la personne âgée et les services formels (Garant et Bolduc, 1990).

Les répercussions de ces tâches se font sentir à plusieurs niveaux : conséquences sur la santé à la suite du stress psychologique, social et physique; frustrations face aux limites placées sur la liberté et le temps de loisir, conflits avec le conjoint et les enfants, et sécurité financière menacée à court et à long terme. Cependant, des aspects de nature plus positive sont également mentionnés par les aidantes et aidants: rencontre de besoins affectifs, besoin d'aider les autres, et apport financier de la personne âgée à la famille. Bref, les personnes qui offrent de l'aide informelle ne font pas que donner, dans cette relation elles reçoivent aussi.

\section{Soutien}

Les études provenant du courant féministe insistent principalement sur le fardeau de l'accompagnement de la personne âgée. 
"Les services le plus souvent suggérés sont les services de répit, d'aide domestique, de repas et de groupes d'entraide.»
Elles recommandent la mise sur pied d'un ensemble de services de soutien (Baines et al. 1991). Les services le plus souvent suggérés sont les services de répit, d'aide domestique, de repas et de groupes d'entraide. Pourtant, même lorsque ces services sont disponibles et que les aidantes et aidants en connaissent l'existence, leur utilisation est beaucoup moins grande qu' on ne l'aurait cru (Kosloski et Montgomery, 1994). En fait, certaines études montrent que les familles préferent offrir elles-mêmes les soins à leurs membres âgés, ou encore ne faire appel qu'à leur propre réseau familial (Lesemann et Martin, 1993).Lorsqu'elles s'adressent finalement aux services formels, les familles sont généralement épuisées et souvent en état de crise (Paquet 1990).

Comment donc expliquer ce dernier phénomène?

\section{Cadre d'analyse}

La majorité des écrits sur la problématique de l'aide informelle font appel à un paradigme positiviste. Dans cette approche, les aidantes et aidants sont perçus comme des objets de recherche de type épidémiologique. Ces personnes subiraient la réalité qui les entoure, réalité qui en fait un groupe à risque ayant besoin de services normalisés, planifiés et livrés par un ensemble de professionnels.

Par contre, le paradigme interprétatif reconnait que la réalité de l'aide informelle est un construit social, et qu'elle varie selon le contexte. Une telle approche, dite anthropologique, cherche alors à cerner le sens que les aidantes et aidants accordent à leur geste et, par le fait même, à saisir les valeurs qui les animent et les soutiennent dans leur façon d'agir. Leur vie de tous les jours échappe ainsi à la planification scientifique (Lesemann et Chaume, 1989). Notre recherche a fait appel à ce deuxième paradigme. 


\section{Résultats}

"On note ainsi non seulement un sens d'obligation envers le conjoint ou le parent, mais également le désir de maintenir le lien avec cette personne le plus longtemps possible.»
Notre étude de l'aide informelle a permis de réaliser qu'il existe deux composantes au geste d'accompagnement. La langue anglaise distingue celles-ci en parlant de caring about et de caring for (Lesemann et Martin, 1993). La première composante évoque la tendresse et la protection maternelle que la famille offre à ses membres âgés, et dont elle n'est pas prête à se départir à moins de ne plus avoir le choix. Nous avons ainsi extrait de notre analyse documentaire et de nos entrevues semi-dirigées les commentaires suivants :

Je veux qu'elle reste à la maison avec moi. Elle est impotente ma pauvre vieille, il faut tout lui faire, mais on est ensemble. (FEACVT 1994: 6)

Pourquoi suis-je resté? Parce que j'aime ma grandmère. Je dois lui rendre ce qu'elle m'a donné. (Koch 1993: 150, notre traduction)

C'est le médecin qui m'a fait comprendre que je n'avais plus le choix. Alors, j'ai dit à mon mari : "Je t'aime, mon vieux, mais j'suis pu capable! Je voudrais tellement pouvoir te garder avec moi, mais j'ai pu les forces qu'il faut!» Mariette - Cliente d'un programme de jour. (Ladouceur, 1995)

On note ainsi non seulement un sens d'obligation envers le conjoint ou le parent, mais également le désir de maintenir le lien avec cette personne le plus longtemps possible. La théorie du don, élaborée par Mauss (1993 [1950]), et reprise aujourd'hui dans les travaux de Godbout et Caillé (1992), est donc apparue comme pouvant contribuer à notre compréhension de ce qui se passe du point de vue des aidantes et aidants.

Pour Mauss, le don a trois mouvements: donner, recevoir et rendre. Il s'agit ici de penser le don non seulement comme un échange, mais un échange qui crée une dette. Cette dernière est 
"...les familles ne demandent pas à l'État de les remplacer... mais demandent plutôt «une aide discrète, complémentaire, ponctuelle plus que permanente, facile d'accès, exempte de valeurs et de normes.» mutuelle, c'est-à-dire que chacun a l'occasion de remettre à l'autre, et par le fait même de créer une autre situation de dette. Ceci a pour effet de maintenir le lien qui, à son tour, assure la solidarité entre individus à travers le temps. Pour comprendre la dynamique de l'aide informelle, la fécondité de la théorie du don permet de saisir ce geste dans un contexte de relations, de solidarités entre les aidants et aidés, alors que l'on reproche tant à nos sociétés modernes d'être caractérisées par l'individualisme (et l'intérêt égoïste).

La deuxième composante de l'aide informelle, soit le caring for, renvoie aux tâches de soutien. Un des aidants interviewés résume assez bien le fardeau que représentent ces tâches:

Je suis un homme à tout faire! C'est ça! Il faut pas rien négliger. La besogne qu'elle faisait, je la fais. La besogne que je faisais, je la fais encore. Pis ça, c'est du matin de six heures à onze heures le soir. Ce sont des journées assez longues. Jean — Entrevue semidirigée. (Ladouceur, 1995)

C'est d'ailleurs davantage pour les tâches de soutien que les familles cherchent à obtenir de l'aide externe.

Ils me demandaient qu'est-ce qui était le plus fatigant pour moi, pour lui donner. Mais j'ai dit «c'est surtout la laver, vous savez, ça c'est..." Fait qu'ils nous envoient une personne deux fois par semaine pour lui donner un bain. (Guberman et al. 1993: 96)

En somme, les familles ne demandent pas à l'État de les remplacer auprès de leurs membres âgés, mais demandent plutôt «une aide discrète, complémentaire, ponctuelle plus que permanente, facile d'accès, exempte de valeurs et de normes» (Godbout et Charbonneau 1994 : 24).

Quelles sont les leçons à tirer de ces résultats? 


\section{Réflexions pour la pratique et les politiques sociales}

"...ce n'est la plupart du temps qu'en dernier recours que les familles font appel aux services publics...» "...la population dans le besoin est la mieux placée pour définir ses besoins..."
Les résultats de notre étude, comme d'ailleurs de plusieurs autres, nous amènent à souligner que l'intervention des professionnels de la santé auprès des aidantes et aidants doit cesser d'être perçue comme menant inévitablement à une prise en charge coûteuse des familles par le système. Ce n'est d'ailleurs pas ce que désirent ces dernières. Au contraire, ce n'est la plupart du temps qu'en dernier recours que les familles font appel aux services publics, préférant s'en tenir à leur propre réseau pour pouvoir mieux préserver le lien avec leurs membres âgés, et leur rendre en même temps l'aide et les soins reçus par le passé. Cette constatation appelle donc les intervenantes et intervenants sociaux à jouer, d'abord, un rôle d'éducation auprès de collègues nouvellement chargés de travailler avec des aidants; il faut aussi agir auprès des décideurs en matière de politiques sociales concernant l'aide informelle, afin que les personnes qui la fournissent reçoivent le soutien nécessaire.

Deuxièmement, les intervenantes et intervenants sociaux se doivent également de participer, avec la population locale, à la mise en place des différentes ressources souhaitées par les aidantes et aidants. Il ne s'agit plus ici de s'en tenir à la validation d'une liste de besoins pré-établis par les chercheurs au sein d'une administration centrale, approche qui pourrait mener à une structure coûteuse de services formels inappropriés. Au contraire, le processus d'analyse de besoins des aidantes et aidants doit plutôt leur laisser la parole. Cette approche inductive, «s'appuie sur le postulat que la population dans le besoin est la mieux placée pour définir ses besoins et la satisfaction de ces derniers" (Mayer et Ouellet $1991: 72$ ). On pourrait ainsi être surpris dans certains cas, de la simplicité des demandes, et de la relative facilité avec laquelle on pourrait répondre à ces besoins au niveau local, avec un appui financier minime de l'État.

Troisièmement, le fait que les familles désirent conserver le plus longtemps possible la responsabilité à l'égard de leurs membres 
"...les intervenants sociaux devraient penser leurs interventions tant au niveau individuel, familial, et de groupe qu'au niveau communautaire..." âgés soulève la nécessité pour les intervenantes et intervenants de jouer un rôle de prévention par rapport à l'épuisement possible chez les aidantes et aidants informels. Cet épuisement peut non seulement miner leur santé, mais peut aussi, dans certains cas, concourir à de la violence envers la personne âgée en perte d'autonomie.

Quatrièmement, il devient important pour les intervenantes et intervenants sociaux de procéder à l'évaluation de leurs propres interventions auprès des aidantes et aidants informels, sinon toute une richesse de connaissances risque de leur échapper sur ce qui fonctionne et ce qui ne fonctionne pas, dans leur approche de cette clientèle. Autant que possible, les intervenantes et intervenants sociaux devraient penser leurs interventions tant au niveau individuel, familial, et de groupe qu'au niveau communautaire, en termes de recherche-intervention (même si ce n'est que sur une base informelle), et avec la participation des aidantes et aidants. Cette approche aurait pour effet de permettre aux intervenantes et intervenants de mieux comprendre les besoins, et de poser à l'avenir des gestes encore plus efficaces.

Enfin, dans les cas où la mise en institution de la personne âgée s'avère incontournable, les intervenantes et intervenants sociaux doivent faciliter les démarches de l'aidante ou l'aidant, afin de maintenir le lien qui l'unit à la personne aidée.

\section{Conclusion}

La plupart des recherches concernant l'aide informelle peignent un portrait plutôt sombre de cette réalité, en parlant surtout de fardeau et d'exploitation. Cependant, l'approche anthropologique cherche, pour sa part, à comprendre la signification que donnent les aidantes et aidants à leur geste. La théorie du don nous aide à comprendre le geste de l'aide informelle, en le situant dans le contexte du lien social qui unit aidants et aidés. On propose des 
"On propose des interventions qui soutiennent les relations qui lient les individus et qui, par conséquent, favorisent les solidarités existantes.» interventions qui soutiennent les relations qui lient les individus et qui, par conséquent, favorisent les solidarités existantes.

Nous espérons avoir pu contribuer, par l'intermédiaire de cet article, à la réflexion qui s'impose dans la pratique et dans les politiques sociales, face au vieillissement de la population. Notre intérêt pour la théorie du don s'inscrit dans un effort de conceptualisation théorique de l'aide informelle, dans le but de repenser le rôle des intervenants et de l'État auprès des aidantes et aidants. Tout un travail d'exploration reste cependant à faire pour bien cerner l'ensemble des implications de cette théorie dans l'étude de l'aide informelle. Nous pensons, entre autres, aux questions déjà soulevées concernant le virage ambulatoire et la promotion du maintien à domicile: s'agit-il d'une exploitation du travail gratuit des femmes, ou d'un désengagement pur et simple de l'État?

\section{Bibliographie}

BAINES, Carol T., Patricia M. EVANS, et Sheila M. NEYSMITH (éds.) (1991). Women's Caring: Feminist Perspectives on Social Welfare, Toronto, McClelland et Stewart.

BRODY, Elaine M. (1990). Women in the Middle: Their Parent-Care Years, New York, Springer.

CANADA. CONSEIL CONSULTATIF NATIONAL SUR LE TROISIÈME ÂGE (CCNTA) (1989). 1989 et Après: Les défis d'une société canadienne vieillissante, Ottawa,Approvisionnements et Services Canada.

FONDATION EUROPÉENNE POUR L'AMÉLIORATION DES CONDITIONS DE VIE ET DE TRAVAIL (FEACVT) (1994). La parole est aux aidants, Dublin, Loughlinstown House.

GARANT, Louise et Mario BOLDUC (1990). L'aide par les proches: mythes et réalités. Québec, Ministère de la Santé et des Services sociaux, Direction de la planification et de l'évaluation.

GODBOUT, Jacques et Alain CAILLÉ (1992). L'esprit du don, Paris, La Découverte

GODBOUT, Jacques T. et Johanne Charbonneau (1994). «Le réseau familial et l'appareil d'état», Recherches sociographiques, vol.XXXV, no 1, 9-38.

GUBERMAN, Nancy, Pierre MAHEU et Chantal MAILLÉ (1993). Et si l'amour ne suffisait pas. Femmes, familles et adultes dépendants, Montréal, Les Éditions du Remue-ménage.

KOCH,Tom (1993). A Place in Time: Care Givers for Their Elderly, Westport (Connecticut), Praeger. KOSLOSKI, Karl et Rhonda J.V. MONTGOMERY (1994). «Investigating Patterns of Service Use by Families Providing Care for Dependent Elders», Journal of Aging and Health, vol. 6 no 1, 17 37.

LADOUCEUR, Linda (1995). Les dynamiques de l'aide informelle auprès des aînées et de aînés. Mémoire de maitrise en service social. Ottawa, Université d'Ottawa. 
LESEMANN, Frédéric et Claire CHAUME (1989). Familles-providence, la part de l'État: Recherche sur le maintien à domicile, Montréal, St-Martin.

LESEMANN, Frédéric et Claude MARTIN (éds.) (1993). Les personnes âgées. Dépendance, soins et solidarités familiales. Comparaisons internationales. Notes et études documentaires no 4967-68, 1993-2-3. Paris, Mission interministérielle de Recherche-Expérimentation.

MAUSS, Marcel (1993[1950]). Sociologie et anthropologie, Paris, Presses Universitaires de France.

MAYER, Robert et Francine OUELLET (1991). Méthodologie de recherche pour les intervenants sociaux, Boucherville, Gaëtan Morin.

PAQUET, Mario (1992). «Le soutien aux aidants qui prennent soin d'une personne âgée en perte d'autonomie», dans Georges Létourneau, éd., Aider ses parents vieillissants: un défi personnel, familial, politique, communautaire, Outremont, Production Immédia, 121-135.

PAQUET, Mario (1990). L'épuisement des familles soutien de personnes âgées: pistes d'intervention pour les professionnels, Communication présentée au Colloque de l'Association québécoise de gérontologie, Région 06, 28 mars 1990. 